\title{
Ring and Peg Electrodes for Minimally-Invasive and Long-Term Sub-scalp EEG Recordings
}

\author{
Y.B. Benovitski ${ }^{1 *}$, A. Lai $^{2 *}$, C.C. McGowan ${ }^{1}$, O. Burns ${ }^{1}$, V. Maxim ${ }^{1}$, D.A.X Nayagam ${ }^{1,3}$, R. \\ Millard $^{1}$, G.D. Rathbone ${ }^{1}$, M.A. le Chevoir ${ }^{6}$, R.A. Williams ${ }^{3}$, D.B. Grayden ${ }^{5,7}$, C.N. May ${ }^{4}$, M. \\ Murphy $^{2}$, W.J. D’Souza ${ }^{2}$, M.J. Cook ${ }^{2}$, C.E. Williams ${ }^{1}$. \\ ${ }^{1}$ The Bionics Institute, East Melbourne, Victoria, Australia. \\ ${ }^{2}$ Department of Medicine, St. Vincent's Hospital, The University of Melbourne, Melbourne, \\ Victoria, Australia. \\ ${ }^{3}$ Department of Anatomical Pathology, The University of Melbourne, St Vincent's Hospital, \\ Melbourne, Australia. \\ ${ }^{4}$ The Florey Institute of Neuroscience and Mental Health, The University of Melbourne, \\ Melbourne, Victoria, Australia. \\ ${ }^{5}$ Department of Electrical and Electronic Engineering, The University of Melbourne, Melbourne, \\ Victoria, Australia. \\ ${ }^{6}$ Faculty of Veterinary and Agricultural Science, The University of Melbourne, Melbourne, \\ Victoria, Australia. \\ ${ }^{7}$ Centre for Neural Engineering, The University of Melbourne, Melbourne, Victoria, Australia. \\ * Equal contributors. \\ Corresponding author at: Bionics Institute, 384-388 Albert St, East Melbourne VIC 3002, \\ Australia. Email address: ybenovitski@bionicsinstitute.org
}

\section{Published in Epilepsy Research - Elsevier} http://dx.doi.org/10.1016/j.eplepsyres.2017.06.003

Received 3 March 2017; Received in revised form 3 May 2017; Accepted 4 June 2017 


\section{Abstract}

Objective

Minimally-invasive approaches are needed for long-term reliable Electroencephalography (EEG) recordings to assist with epilepsy diagnosis, investigation and more naturalistic monitoring. This study compared three methods for long-term implantation of sub-scalp EEG electrodes.

Methods

Three types of electrodes (disk, ring, and peg) were fabricated from biocompatible materials and implanted under the scalp in five ambulatory ewes for 3 months. Disk electrodes were inserted into sub-pericranial pockets. Ring electrodes were tunneled under the scalp. Peg electrodes were inserted into the skull, close to the dura. EEG was continuously monitored wirelessly. High resolution CT imaging, histopathology, and impedance measurements were used to assess the status of the electrodes at the end of the study.

Results

EEG amplitude was larger in the peg compared with the disk and ring electrodes $(\mathrm{p}<0.05)$. Similarly, chewing artifacts were lower in the peg electrodes $(p<0.05)$. Electrode impedance increased after long-term implantation particularly for those within the bone $(p<0.01)$. Micro-CT scans indicated that all electrodes stayed within the sub-scalp layers. All pegs remained within the burr holes as implanted with no evidence of extrusion. Eight of 10 disks partially eroded into the bone by $1.0 \mathrm{~mm}$ from the surface of the skull. The ring arrays remained within the sub-scalp layers close to implantation site. Histology revealed that the electrodes were encapsulated in a thin fibrous tissue adjacent to the pericranium. Overlying this was a loose connective layer and scalp. Erosion into the bone occurred under the rim of the sub-pericranial disk electrodes.

\section{Conclusions}

The results indicate that the peg electrodes provided high quality EEG, mechanical stability, and lower chewing artifact. Whereas, ring electrode arrays tunneled under the scalp enable minimal surgical techniques to be used for implantation and removal.

\section{Keywords}

Long-term EEG; Epilepsy; Sub-scalp electrode; Minimally-invasive; Stable; Removable. 


\section{Introduction}

Around 50 million people worldwide have epilepsy (World Health Organization, 2012) and 30$40 \%$ of these patients are drug resistant (Kwan \& Sander, 2004). One study estimated that 92,000 people were misdiagnosed with epilepsy in England and Wales in 2002 and were inappropriately prescribed anti-epileptic drugs (Juarez-Garcia, Stokes, Shaw, Camosso-Stefinovic, \& Baker, 2006). The side effects of anti-epileptic drugs include cardiovascular complications (Vyas et al., 2015), cognitive and behavioral deficits (Ortinski \& Meador, 2004), and other adverse reactions (Bainbridge \& Oh, 2013). These diagnostic and therapeutic issues put patients at significant risk and cause a substantial burden on the healthcare system (Juarez-Garcia, et al., 2006).

The most common diagnostic instruments for epilepsy involve the analysis of EEG signals recorded from scalp electrodes. These are useful for short-term recordings but are not currently suitable for long-term monitoring indications. Moreover, blackouts are often misdiagnosed as having an epileptic cause (Benbadis, 2009; Petkar, Cooper, \& Fitzpatrick, 2006; Smith, 2001) because these events are often infrequent (weeks to months apart) and can be situational, so it is difficult to capture them with in-patient EEG monitoring.

Earlier studies indicate a need for a long-term implantable device to continuously monitor epileptiform events to aid clinical management of epilepsy patients (for review see Freestone et al., 2015). A recent long-term clinical trial of a seizure advisory system with electrodes implanted under the skull showed that entries in patient diaries often do not correspond with recorded epileptiform events (Cook et al., 2013). This study used epidural electrodes that require craniotomy. Other surgical approaches, used for acute epileptogenic zone identification, involve subdural and depth electrodes (for review see Sperling, 1997). However, these are highly invasive and are not suitable for long-term monitoring of epilepsy in a wider patient population, especially in ambulatory setting, so a less invasive approach is required. Sub-scalp electrodes may provide an optimal solution for minimally-invasive, long-term epilepsy monitoring.

The main objective of the current study was to assess the feasibility of three types of sub-scalp electrodes for long-term implantation. These were developed to suit three different sub-scalp surgical approaches: peg electrodes inserted into burr holes in the cranium, disk electrodes slid into pockets created under the pericranium, and ring electrodes tunneled under the scalp via a small guide cannula. Electrodes have been previously placed in burr holes (Barnett, Burgess, Skipper, Edwards, \& Luders, 1990; Holthausen, Nochtar, Pannek, Ebnar, \& Tuxhorn, 1994; Williams, Gunn, Synek, \& Gluckman, 1990) but not tunneled under the scalp such as the ring and disk. Compared to earlier versions, electrodes fabricated for this study are fixed in size. This approach allows the implant to be pre-assembled, reducing surgical complexity and improving reliability.

The aim of this project was to compare long-term signal quality of the EEG, surgical feasibility, and stability of these three types of electrodes in sheep. Sheep were used in this study because 
their skull thickness (6 vs. 6.3mm; Laure, Petraud, Sury, Tranquart, \& Goga, 2012) and dimensions are comparable to humans.

\section{Material and methods}

\subsection{Subjects}

This research was approved by the Florey Institute of Neuroscience and Mental Health Animal Ethics Committee, AEC Number 14-108-FINMH, that adheres to the Australian Code for the Care and Use of Animals for Scientific Purposes.

Five healthy Merino ewes, 1.5-2 years old (sheep S1 to S5) were used for this study. Sheep were individually housed in $12 \mathrm{~h}$ light/dark cycle and given free access to water and fed oaten chaff once a day. Food and water intake and the well-being of the animals were monitored daily.

\subsection{Implant}

Three types of electrodes, peg, disk, and ring, were fabricated at the Bionics Institute from biocompatible silicone and stainless steel (Figure 1). They were designed for sub-scalp placement procedures and removability. The surface areas of the three types of electrodes were similar at about $\sim 32 \mathrm{~mm}^{2}$, which resulted in comparable saline impedance. Peg electrodes had a progressive taper to prevent bone encapsulation and promote ease of removal.

They were connected to a fully-implantable transmitter (PhysioTel model D70-EEE, $455 \mathrm{kHz}$, input voltage range $\pm 2.5 \mathrm{mV}$, and channel bandwidth 1-100 Hz from Data Sciences International, DSI, New Brighton, MN, USA) used to transmit EEG signals to a DSI external data logger. All DSI equipment was factory calibrated. Pairs of peg and disk electrodes were individually attached and the two ring electrodes were connected as an array (Figure 2). Implants were assembled in a clean room, tested for impedance, and cold sterilized prior to implantation. Implants had fixed dimensions and came in one piece so no additional assembly was required during implantation.

The first animal was implanted with an earlier version that did not have the two ring electrodes thus the total number of electrodes was 28 .

\subsection{Implant Surgery}

Electrodes and transmitter were implanted under general anesthesia using standard sterile surgery techniques. Anesthesia was induced with intravenous sodium thiopental $(15 \mathrm{mg} / \mathrm{kg})$ and 
ongoing intubation was maintained with 1.5-2.0\% isoflurane/O2. Sheep were treated with intramuscular antibiotics (900 mg, procaine penicillin, Troy Laboratories, NSW) at surgery and 2 days post-operatively. Analgesia was maintained with intramuscular flunixin meglumine (1 $\mathrm{mg} / \mathrm{kg}$; Troy Laboratories) at surgery and $4 \mathrm{~h}$ post-surgery. Reflexes were tested to ensure adequate anesthetic and vital signs were recorded throughout the procedure. Sheep were placed in a stereotaxic frame and electrodes were implanted via a midline incision. The soft tissue was separated from the skull on the right side using hemostats to form a skin "flap". The anatomical targets are illustrated in Figures 2 and 3.

For the disk electrodes, a periosteal elevator was used to create a sub-pericranial pocket for each disk. The first disk was positioned $1.5 \mathrm{~cm}$ from midline and the second disk $2 \mathrm{~cm}$ posteriorly to the first.

For the ring electrode, a small, custom-made guide cannula was tunneled under the scalp through to the open flap. The cannula tip was removed and the ring electrode array was inserted into the cannula so it could be positioned under the scalp and the cannula was removed.

For the peg electrode, a periosteal elevator was used to clear the location of the burr hole. A 3.1 mm diameter round cutting burr was used to drill to the lower table until the dura was just visible. The wall of the burr hole was widened using a handheld $4.5 \mathrm{~mm}$ diameter reamer for the silicone overmolding to fit. Depth and size of burr hole were checked for fit using silicone dummy electrodes. Each electrode was inserted into the hole and rotated so that the leads had the right amount of tension and minimum slackness. The first peg was positioned $7 \mathrm{~mm}$ from the midline suture and $16 \mathrm{~mm}$ anterior from bregma and the second peg $6 \mathrm{~mm}$ posterior to bregma/occipital suture.

Leads were tunneled under the skin so that the telemetry device could be placed into the pocket in the neck. Following implantation of the electrodes, the skin was brought together and sutured.

\subsection{EEG Recordings}

EEG recordings were made over 10 weeks for all five animals and all 28 implanted electrodes. This allowed assessment of signal quality and stability over time. Sheep were videotaped to document movements. Recordings were made simultaneously from three electrode pairs at a sampling rate of $500 \mathrm{~Hz}$. The rostral electrodes were likely covering the parietal cortex and the caudal ones the occipital cortex. These regions are involved in cognitive association. 


\subsection{EEG Signal Processing}

Recordings were imported into proprietary EEG software (Persyst, San Diego, CA, USA) for amplitude-integrated electroencephalography (aEEG), and fast Fourier transform (FFT) spectrogram analysis. The aEEG relies on the specifications of the original cerebral function monitor (Maynard, Prior, \& Scott, 1969). Time course measurements were binned as follows: $0.4-0.9,0.9-3,3-5$ and 10-35 weeks.

Using the filters and the spectrogram function in Persyst, high amplitude slow wave long duration epochs (below 15Hz and over 15-20 minutes), short duration high amplitude slow wave (below $15 \mathrm{~Hz}$ and over 1-2 minutes without chewing or any other artifacts), chewing (muscle activity bursts with $\sim 1$ second interval), and chewing and slow wave (occurring simultaneously) segments were marked (Figure 5 shows representative examples). FFT spectrograms were stable during each segment. Data were then imported into MATLAB (Versions 7.14 and 9.0, MathWorks, Natick, MA, USA) for further processing and statistical analysis.

Chewing artifact index was defined as the ratio between slow wave EEG and chewing artifact amplitude. Chewing artifact (noise) and slow wave (signal) were extracted by $30 \mathrm{~Hz}$ cut-off high-pass and low-pass filters, respectively. When a simultaneous slow wave and chewing segment was not available, data segments containing those waveforms separately were assessed.

The EEG measurements were compared between the three different electrodes with nonparametric methods, Friedman chi-square and Kruskal-Wallis with Bonferroni correction. Data were analyzed across all 28 electrodes and presented as median (upper and lower quartile) for each electrode type. Statistical significance levels below 0.05 and 0.01 were considered.

\subsection{Imaging and histology}

At the completion of the EEG recordings (3-6 months), animals were anesthetized and euthanized with Lethobarb i.v (100mg/kg, Virbac, NSW, Australia). The head was perfused via the carotid arteries with warm $\left(37^{\circ} \mathrm{C}\right)$ heparinized (DBL Heparin sodium BP, $0.1 \%$, Hospira Aust. Pty. Ltd., Victoria, Australia) $0.9 \%$ saline followed by cold $\left(4^{\circ} \mathrm{C}\right) 10 \%$ neutral buffered formalin (NBF) (Sigma-Aldrich, NSW, Australia). Tissue was then collected for histological analysis to assess the tissue reaction and safety of the chronically implanted electrodes. The samples containing the electrodes were then post-fixed in $10 \% \mathrm{NBF}$ at $4{ }^{\circ} \mathrm{C}$ until micro-computed tomography (Micro-CT) scans were performed.

Skull samples were scanned using a SkyScan 1076 micro-CT scanner (Bruker, Kontich, Belgium). Before scanning, samples were wrapped in gauze saturated with $70 \%$ ethanol and placed inside an enclosed plastic container to prevent them from drying. Images were obtained using $100 \mathrm{kV}, 100 \mu \mathrm{A}, 440 \mathrm{~ms}$ exposure at $18 \mu \mathrm{m}$ spatial resolution with $0.1^{\circ}$ rotation steps for a 
full $360^{\circ}$ rotation, which resulted in 3600 projection images. Beam hardening artifacts were minimized by applying the scanner's thickest filter ( $1.0 \mathrm{~mm}$ aluminum filter). Frame averaging was set at 6 for noise reduction on the images. Scanned data were reconstructed by Skyscan NRecon (Bruker) software employing no smoothing, a 30\% beam hardening correction, a ring artifact reduction value of 7 , and post-alignment values calculated by NRecon. The histogram limits were set by referring to the reconstructed preview image to get good visible contrast between the bone and metallic electrode (a minimum of 0 Hounsfield Units (HU) and a maximum of typically $10000 \mathrm{HU}$, ranging from 8000 to $13500 \mathrm{HU}$ ).

Distances from the electrode surface to the inner surface of the skull were measured using Skyscan's Data Viewer software. Distance was measured in nine, five, and one equidistant locations for the disk, ring, and peg electrodes, respectively. Multiple locations were measured to account for variability in skull thickness.

Skull and scalp tissue samples were then decalcified in $10 \%$ Ethylenediaminetetraacetic acid (Sigma-Aldrich, NSW, Australia) for 3-6 months. The electrodes were removed prior to processing. Decalcified tissue samples were trimmed $(2 \mathrm{~cm} \times 2 \mathrm{~cm})$ and embedded in paraffin wax. Paraffin-embedded tissue sections were sliced $5 \mu \mathrm{m}$ thick and mounted on superfrost slides (Grale scientific, Victoria, Australia). Routine Haematoxylin and Eosin (H\&E) staining was performed. Sections were imaged using Axio Imager 2 upright microscope (Carl Zeiss).

\subsection{Impedance Measurement}

Impedance measurements were conducted using a potentiostat (Gamry Instruments, Z100, Interface 1000), for each electrode using $1 \mathrm{kHz}$ stimulus at the end of the chronic implantation period during the post-mortem analysis and compared with impedance measured in saline. Kruskal-Wallis with Bonferroni correction analysis was also used to compare impedance of all implanted electrodes.

\subsection{Removability}

Removability of four passive ring electrode arrays (eight electrodes) was assessed at postmortem after being chronically implanted subcutaneously in the neck. Maximum force required to pull the electrode arrays was recorded using a digital force gauge (Mark-10 Corporation, sensitivity: $0.01 \mathrm{~N})$. 


\section{Results}

Micro-CT scans indicated that all 28 electrodes stayed within the sub-scalp layers. All pegs remained within the burr holes as implanted with no evidence of extrusion. Eight disks partially eroded into the bone by $1.0 \mathrm{~mm}(0.82 \mathrm{~mm}$ and $1.1 \mathrm{~mm}$ quartiles $)$ from the surface of the skull. All the ring arrays remained within the sub-scalp layers close to where they were implanted. A representative example of each electrode scan is shown in Figure 4.

The transmitter pocket in the neck in two animals required attention as the device was situated directly under the suture line.

Figure 5 shows representative examples of EEG activity for all electrodes in one sheep recorded during slow wave EEG and chewing. Peg electrode had higher EEG amplitude and lower chewing artifact. Long-term aEEG measurements were stable from all five animals (S1 to S5) over 3 months with non-significant subject and time effects (Friedman chi-square, p>0.05) as shown in figure 6.

Figure 7 shows aEEG for all sheep plotted for each electrode type. EEG amplitude of the pegs was larger with respect to disks and rings. Kruskal-Wallis with Bonferroni indicated significant differences in aEEG between electrodes: $p<0.05$ for disk and ring; $p<0.01$ for the peg.

Figure 8 shows chewing artifact index for all sheep for each electrode type. This index is the ratio between median amplitude of slow wave EEG and chewing artifact. Kruskal-Wallis with Bonferroni correction (75 degrees of freedom) indicated significant differences in chewing artifact index between electrodes: $p<0.05$ for disk and ring; $p<0.01$ for the peg.

Impedance results are shown in Table 1. Impedance in saline was similar across the three electrode types (139.7 $\Omega$ median and $132.9 \Omega$ lower, $143.5 \Omega$ upper quartiles). Impedance measurements at the end of the experiment were increased in vivo compared to in saline. Specifically, an increases of about 3-, 4.8-, and 6- fold were measured for rings, disks, and pegs respectively. Comparing impedances of all 28 implanted electrodes, Kruskal-Wallis with Bonferroni correction showed differences between the ring and other two electrode types $(p<0.01)$. Measurements for two of the ten pegs were very high indicating a lead fault at the end of the experiment. As there were no significant changes in the analyzed EEG over time, it was determined that the lead faults occurred after the EEG recording period.

Distances between each of the 28 electrodes measured to the inner surface of the skull and median across five animals with lower and upper quartile were taken. The tip of the pegs was $0.69 \mathrm{~mm}$ (0.32 and 1.24 quartiles). Median distance for the disk in nine locations was $4.06 \mathrm{~mm}$ (3.28 and 5.97 quartiles). Median distance for the rings in five locations was $4.86 \mathrm{~mm}$ (3.55 and 6.49 quartiles). 
After fixation and imaging, electrodes were removed from the tissue before histological processing. The ring electrodes leads could be easily withdrawn, in contrast to the peg and disk electrodes, which required surgical exposure before they could be removed from the fibrotic capsule. The passive ring electrode arrays could be removed with a median pull force of $0.62 \mathrm{~N}$ (0.61 lower and 0.67 upper quartiles, $\mathrm{n}=4$ ).

Overall, the histopathological responses at implantation site were unremarkable compared with distal tissue. The electrodes were encapsulated in a thin fibrous capsule with minimal inflammation. Superior to this was a loose fibrous layer and then the upper layers of the scalp. Inferior to the peg electrodes, there was bone with fibrous tissue in some of the voids. Downgrowth of pericranium-like connective tissue was observed on the inner wall of the electrode cavity (Figure 9). There was histological evidence of bone erosion under the rim of the disks (Figure 10). The region surrounding a ring electrode cavity was also surrounded by a thin fibrous capsule partially lined by histiocytic cells (Figure 11).

\section{Discussion}

Potentially implantable long-term monitoring techniques could be complementary to widely used short-term scalp EEG diagnostics. This study compared three different electrodes for sub-scalp electrode implantation: peg, disk, and ring electrodes. The purpose of this was to determine feasibility for long-term epilepsy monitoring indications.

\subsection{Surgical approach}

A key ring electrode advantage is that multiple electrodes and the lead can be tunneled under the scalp via small incision (single guide cannula insertion). In contrast, the peg and disk electrodes require larger incision in addition to tunneling of the leads. The former approach will reduce the number and size of incisions with lower risk of wound complications.

The results indicate that enhanced signal quality can be obtained from pre-assembled (fixed height) peg electrodes, chronically implanted into burr holes as long as they are shorter than the minimum skull thickness. Fixed size electrodes and a pre-assembled implant can reduce surgical complexity and thus the risk of infection. This contrasts with the acute use of peg electrodes where the size of each peg is individually matched to the depth of the burr holes (Barnett, et al., 1990; Holthausen, et al., 1994).

The peg electrodes offer an additional benefit in that they can be rotated to accommodate excess lead length and provide strain relief. This was not possible with the disk electrodes as they were 
placed in sub-pericranial pockets and could not be rotated. There may be constraints in the creation of pockets close to bone sutures and with increased age of the patient. Furthermore, the peg electrodes do not require anchoring (additional suturing) compared with other electrode types.

\subsection{Stable long-term EEG monitoring}

Stable long-term EEG was achieved with the three types of sub-scalp electrodes. Peg electrodes that are closer to the surface of the brain had higher EEG amplitude compared to the disk and ring electrodes. As expected, EEG amplitude increased as distance between the electrode and the brain reduced. These results are comparable to clinical recordings with larger amplitudes for subdural relative to scalp EEG.

\subsection{Muscle and chewing artifacts}

There are significant issues with scalp EEG monitoring particularly when close to the temporalis muscles. In this study, sub-scalp ring and disk electrodes were susceptible to these artifacts. The prominent distribution of muscles in the sheep means that chewing artifact was significant across the scalp for the disks and rings. Clinically, electrodes can be placed distal to temporalis muscles to minimize this artifact. In contrast, the pegs showed minimal chewing artifact. This was in part due to the higher amplitude of the EEG signal. Pegs may, for example, be advantageous for placement under the temporalis muscle for recording EEG from the temporal lobe.

\subsection{Long-term implantation}

Broadly, these electrodes were well tolerated for long-term implantation and remained in the sub-scalp placement. The three electrode types were encapsulated in a thin fibrous capsule that was generally contiguous with the pericranium and surface of the skull. The scalp remained above a layer of loose tissue without apparent adhesions. The bone grew close to the surface of the pegs in the burr hole but there was no evidence of extrusion.

Eight of the ten sub-pericranial disks partially eroded into the skull. The reason for this is not clear but may have resulted from localized mechanical pressure from the rim of the disks and overlying pericranium. However, this is surprising as the rim was over-molded in soft silicone. The isolation of the bone from the pericranium may have sensitized this erosion. Furthermore, some of the electrode leads eroded through the scalp where there were loops in their placement. This lead loop issue needs to be avoided in clinical applications. 


\subsection{Removability}

Ease of removability differed between the three electrode types. Ring arrays were pulled out with minimal force and without surgical exposure of the electrode site, thus they were the easiest to remove. Disk electrodes were encapsulated in thin fibrous pockets but, due to their shape, required surgical exposure at the electrode site. Peg electrodes also required surgical exposure at the electrode site so they could be easily removed from the bone as they had a progressive taper that prevented bone encapsulation.

\subsection{Electrode impedance}

Impedance measurements at the end of the experiment were 3-, 4.8-, and 6-fold higher for rings, disks, and pegs respectively. This difference in rise can be explained by the relative amount of tissue and bone encapsulation for each electrode type. The peg impedance was highest due to increased bone contact.

\subsection{Spatial resolution}

The intended usage of the sub-scalp electrodes is seizure counting and not source localization, so implanting electrodes on both sides of the hemisphere to determine which side is involved in each seizure is likely to be sufficient. It is possible to implant multiple ring electrodes to get a large coverage with the same spatial resolution as the International 10-20 EEG system, but the surgery would be much more invasive.

\subsection{Conclusion}

This study demonstrates stable long-term EEG monitoring using 3 types of sub-scalp electrodes in sheep. The ring electrodes enable mapping of traditional EEG geometry with the advantage of less invasive implantation and removal procedures. Disk electrodes are contraindicated due to risk of erosion into the skull. In contrast, the peg electrodes provided mechanical stability and a higher quality signal in the presence of chewing artifacts. These may be useful for specific longterm diagnostic or monitoring indications for epilepsy patients. 


\section{Conflict of interest statement}

None of the authors have any conflict of interest to disclose. We confirm that we have read the Journal's position on issues involved in ethical publication and affirm that this report is consistent with those guidelines.

\section{Acknowledgements}

The authors acknowledge the support from the Australian National Health and Medical Research Council Project Grant (APP1075347). The Bionics Institute acknowledges the support it receives from the Victorian Government through its Operational Infrastructure Support Program. The funding sources had no involvement in study design, collection, analysis and interpretation of data, writing of the report, or the decision to submit the article for publication.

The authors would like to thank Tony Dornom, Tom Vale, Alan McDonald, and Anne Coco for providing animal care and help with surgeries, Dr. Tom Oxley for providing access to equipment and Prof. Peter Seligman for advice and manuscript review. Authors would also like to thank Shelley O'Hara, Marvin Lai and Peter Brotchie for help with CT imaging and Dr. Cesar M. Salinas-La Rosa for reviewing histology results. 


\section{References}

Bainbridge, J. L., \& Oh, C. Y. (2013). Antiepileptic Drug Adverse Effects: What to Watch Out For. In J. W. Miller \& H. P. Goodkin (Eds.), Epilepsy (1 ed., pp. 84 - 90). Somerset, GB: Wiley-Blackwell.

Barnett, G. H., Burgess, R. C., Skipper, G. J., Edwards, C. R., \& Luders, H. (1990). Epidural peg electrodes for the presurgical evaluation of intractable epilepsy. Neurosurgery, 27(1), 113-115.

Benbadis, S. (2009). The differential diagnosis of epilepsy: a critical review. Epilepsy \& Behavior, 15(1), 15-21.

Cook, M. J., O'Brien, T. J., Berkovic, S. F., Murphy, M., Morokoff, A., Fabinyi, G., . . Himes, D. (2013). Prediction of seizure likelihood with a long-term, implanted seizure advisory system in patients with drug-resistant epilepsy: a first-in-man study. The Lancet Neurology, 12(6), 563-571. doi: 10.1016/s1474-4422(13)70075-9

Freestone, D. R., Karoly, P. J., Peterson, A. D., Kuhlmann, L., Lai, A., Goodarzy, F., \& Cook, M. J. (2015). Seizure Prediction: Science Fiction or Soon to Become Reality? Current Neurology and Neuroscience Reports, 15(11), 1-9.

Holthausen, H., Nochtar, S., Pannek, H., Ebnar, A., \& Tuxhorn, I. (1994). Foramen ovale and epidural peg electrodes. Acta Neurologica Scandinavica, 89(S152), 39-43.

Juarez-Garcia, A., Stokes, T., Shaw, B., Camosso-Stefinovic, J., \& Baker, R. (2006). The costs of epilepsy misdiagnosis in England and Wales. Seizure, 15(8), 598-605.

Kwan, P., \& Sander, J. (2004). The natural history of epilepsy: an epidemiological view. Journal of Neurology, Neurosurgery \& Psychiatry, 75(10), 1376-1381.

Laure, B., Petraud, A., Sury, F., Tranquart, F., \& Goga, D. (2012). Resistance of the sheep skull after a monocortical cranial graft harvest. Journal of Cranio-Maxillofacial Surgery, 40(3), 261-265.

Maynard, D., Prior, P. F., \& Scott, D. (1969). Device for continuous monitoring of cerebral activity in resuscitated patients. British medical journal, 4(5682), 545.

Ortinski, P., \& Meador, K. J. (2004). Cognitive side effects of antiepileptic drugs. Epilepsy \& Behavior, 5, 60-65.

Petkar, S., Cooper, P., \& Fitzpatrick, A. P. (2006). How to avoid a misdiagnosis in patients presenting with transient loss of consciousness. Postgraduate medical journal, 82(972), 630-641.

Smith, P. E. (2001). If it's not epilepsy. Journal of Neurology, Neurosurgery \& Psychiatry, 70(suppl 2), ii9ii14.

Sperling, M. R. (1997). Clinical challenges in invasive monitoring in epilepsy surgery. Epilepsia, 38(s4), S6S12.

Vyas, M. V., Davidson, B. A., Escalaya, L., Costella, J., Saposnik, G., \& Burneo, J. G. (2015). Antiepileptic drug use for treatment of epilepsy and dyslipidemia: systematic review. Epilepsy research, 113, 44-67.

Williams, C. E., Gunn, A. J., Synek, B., \& Gluckman, P. D. (1990). Delayed seizures occurring with hypoxicischemic encephalopathy in the fetal sheep. Pediatr Res, 27(6), 561-565. 


\section{Figures and Tables}

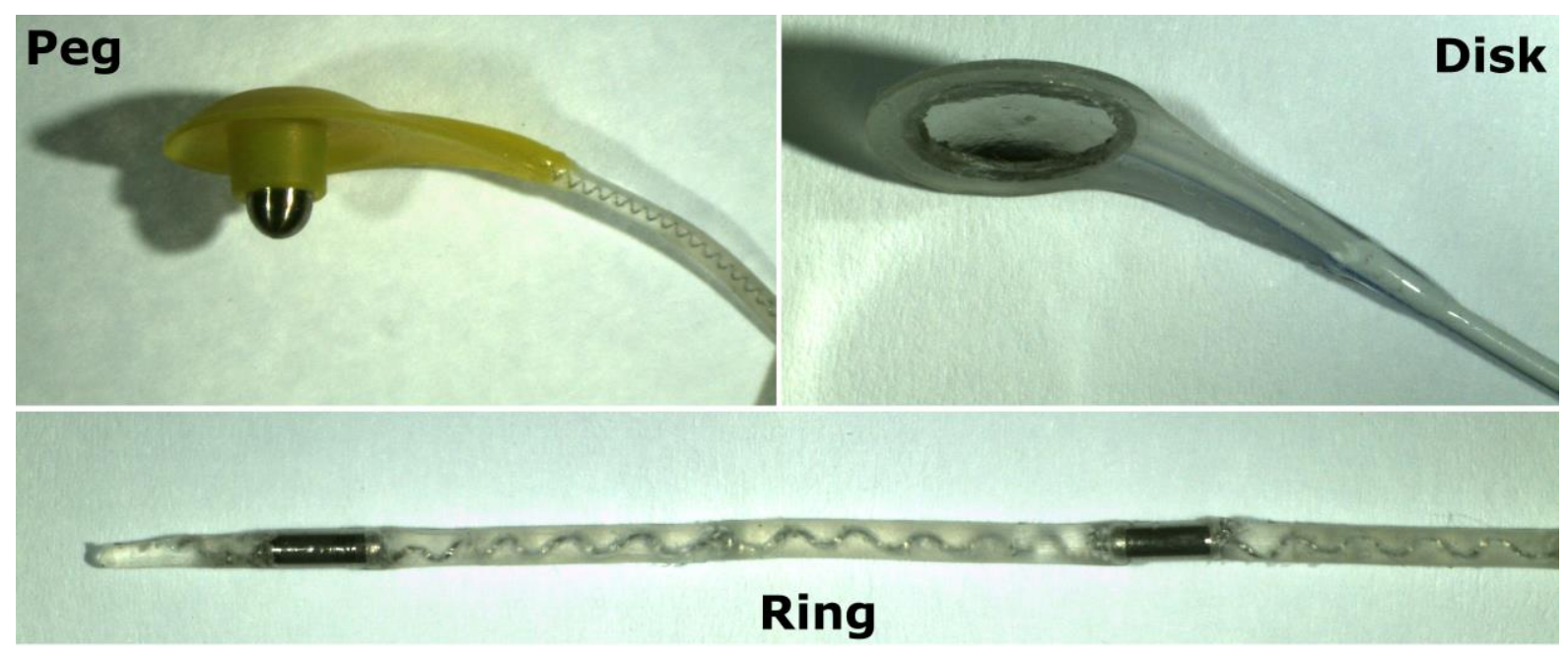

\section{Figure 1}

Photographs of the manufactured electrodes, ring, disk, and peg before implantation. Surface areas of the three electrode types were similar at about $\sim 32 \mathrm{~mm}^{2}$. 


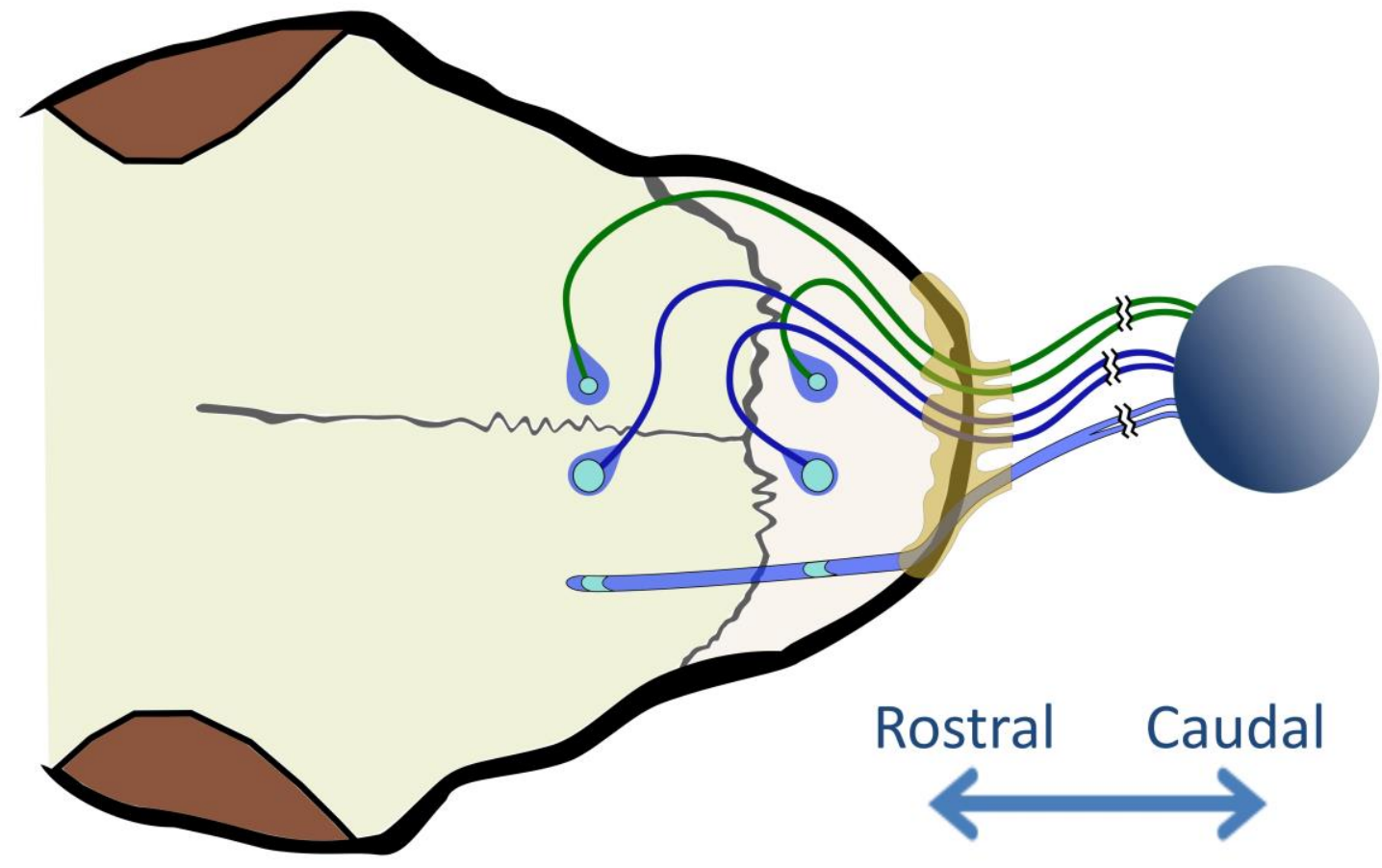

\section{Figure 2}

Illustration of the implantable system guide. Pairs of disk, peg and ring electrodes are represented from top to bottom, respectively. Location of the placement of the sub-scalp electrodes is in respect to coronal and sagittal (midline) sutures. Brown marks (most rostral) are the orbital sockets; beige mark (caudal to electrodes) is silicone patch providing anchoring. DSI transmitter (60 mm diameter) is placed away under the skin of the neck.

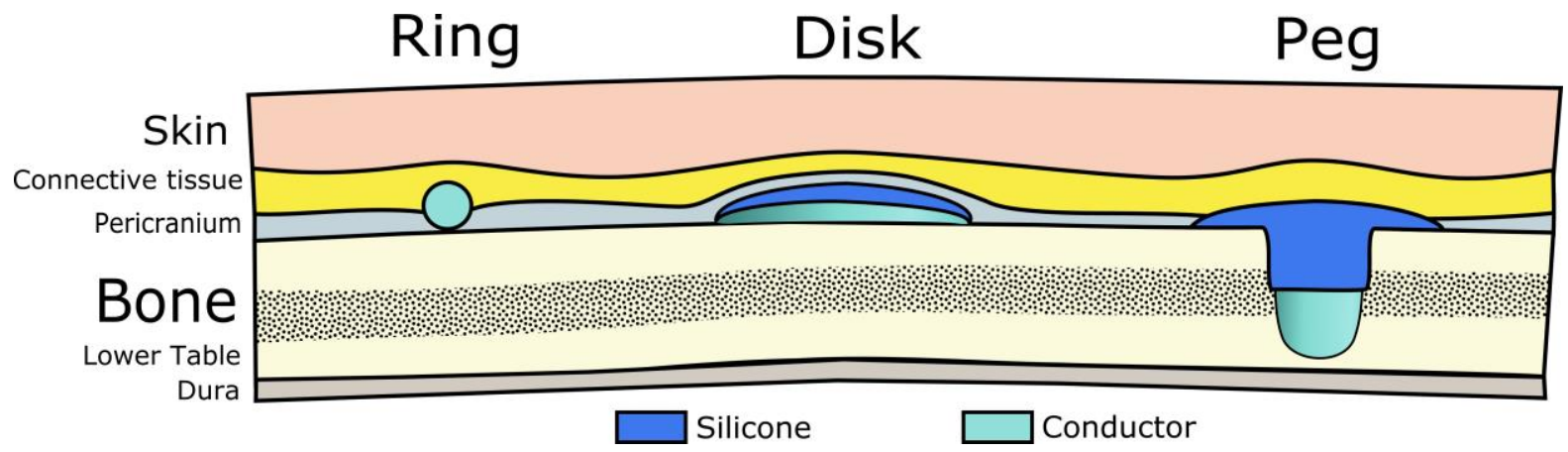

\section{Figure 3}

Surgical position placement of the electrodes within the layers of the scalp. Disk electrodes were inserted contralateral to the peg electrodes in pockets under the pericranium. Ring electrodes were tunneled in the cleavage plane between the aponeurosis and the pericranium. Peg electrodes 
were implanted in burr holes within the right side parietal bone. Disk and peg electrodes are overmolded in soft, biocompatible silicone. 

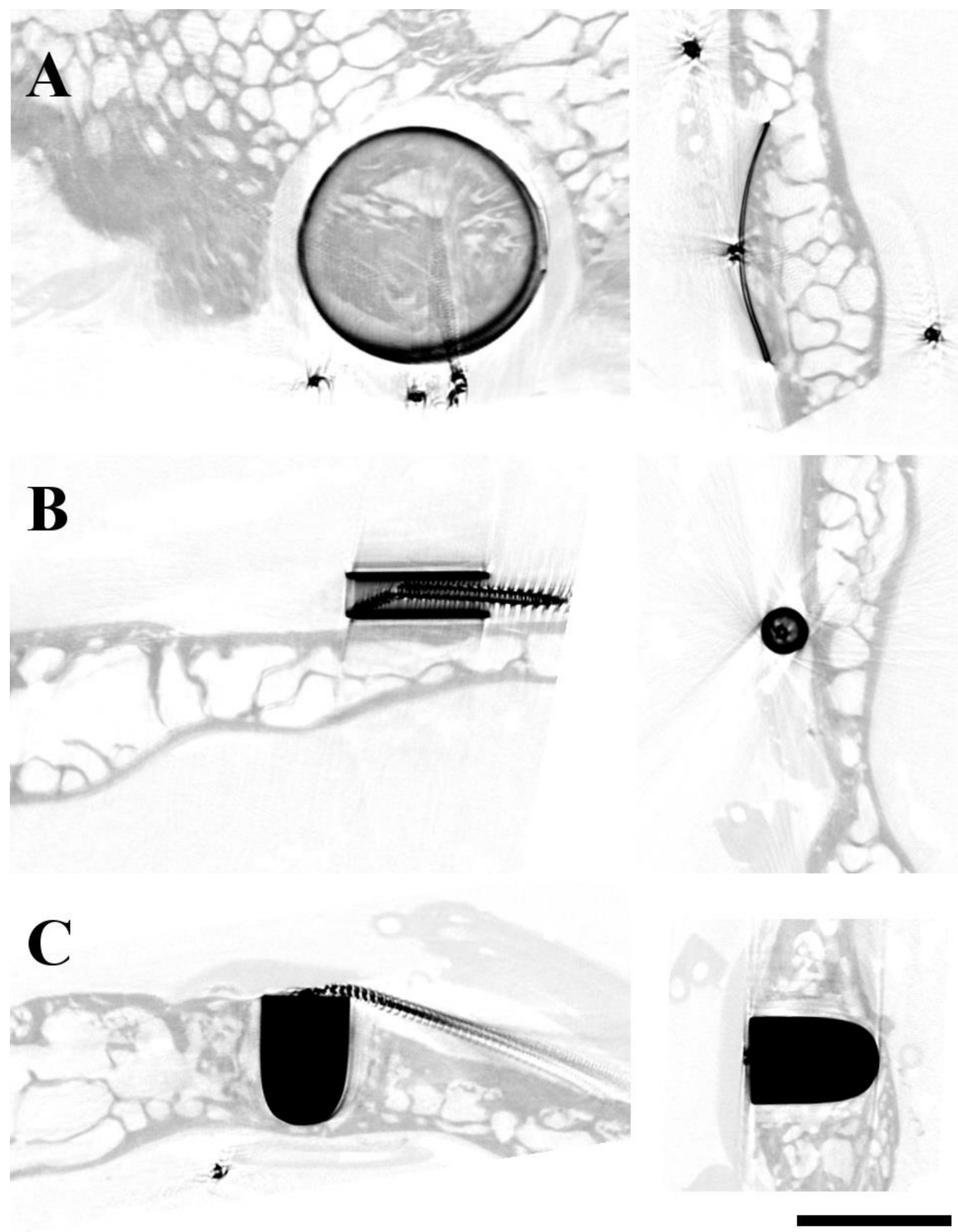

Figure 4

Micro-CT scans show representative examples of implanted electrodes. Eight out of ten disks partially eroded into the bone as seen in this example (A). The ring electrodes were seen on or 
close to the surface of the skull (B). The surface of the peg remained adjacent to the surface of the skull as implanted. The tips of the peg electrodes were closest to the dura positioned in the lower table of the skull. Bone within the burr hole was adjacent to the surface of the electrode and silicone overmolding $(\mathrm{C})$. Scale bar $=5 \mathrm{~mm}$. 


\begin{tabular}{cccccccc} 
& Ring (+) & Ring (-) & Disk $(+)$ & Disk $(-)$ & Peg $(+)$ & Peg $(-)$ & Reference \\
\hline S1 & n/a & n/a & 474.3 & 544.8 & 563.3 & o/c & 85.5 \\
S2 & 433.4 & 293.8 & 616.7 & 669.0 & 704.3 & 895.7 & 89.4 \\
S3 & 510.5 & 362.7 & 616.0 & 993.3 & 1241.0 & 1397.0 & 92.1 \\
S4 & 444.6 & 324.9 & 747.0 & 817.2 & 903.8 & o/c & 169.8 \\
S5 & 289.8 & 317.5 & 660.3 & 981.0 & 720.1 & 868.0 & 155.0 \\
\hline Saline & 137.1 & 111.6 & 142.3 & 131.6 & 152.0 & 143.9 &
\end{tabular}

\section{Table 1}

Impedance in Ohm for all 28 implanted electrodes measured in vivo at the end of the experiment using $1 \mathrm{kHz}$ stimulus and impedance measured in saline. Note that $\mathrm{n} / \mathrm{a}$ indicates not available recordings and o/c indicates open circuit. Impedance in saline was similar across the three electrode types (139.7 $\Omega$ median and $132.9 \Omega$ lower $143.5 \Omega$ upper quartiles). In vivo impedance increased compared with in saline. Specifically, an increase of about 3, 4.8, and 6 fold was measured for rings, disks, and pegs respectively. Comparing impedance of all 28 implanted electrodes, Kruskal-Wallis with Bonferroni correction showed difference between the ring and other two electrode types $(\mathrm{p}<0.01)$ and nonsignificant difference between disk and peg $(\mathrm{p}>0.4)$. Measurements for 2 out of 10 pegs were very high indicating a lead fault at the end of the experiment. As there were no significant changes in the analyzed EEG over time, it was determined that the lead faults occurred after the period of EEG recording. 

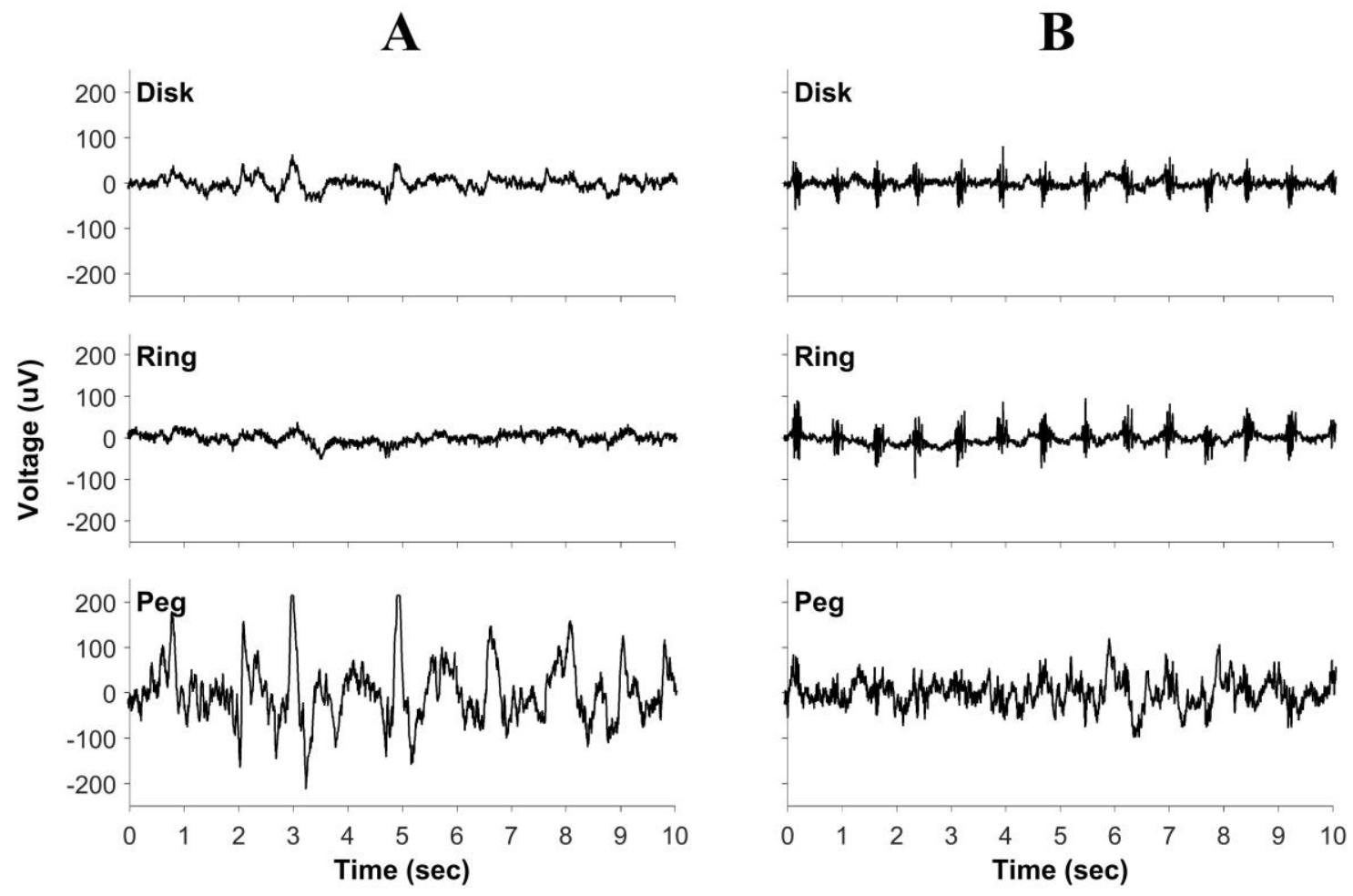

\section{Figure 5}

Representative examples of EEG activity from disk, ring, and peg sub-scalp electrodes for sheep S2, day 31, slow wave (A), chewing and slow wave (B). Peg electrode has higher signal amplitude and lower chewing artifact. 

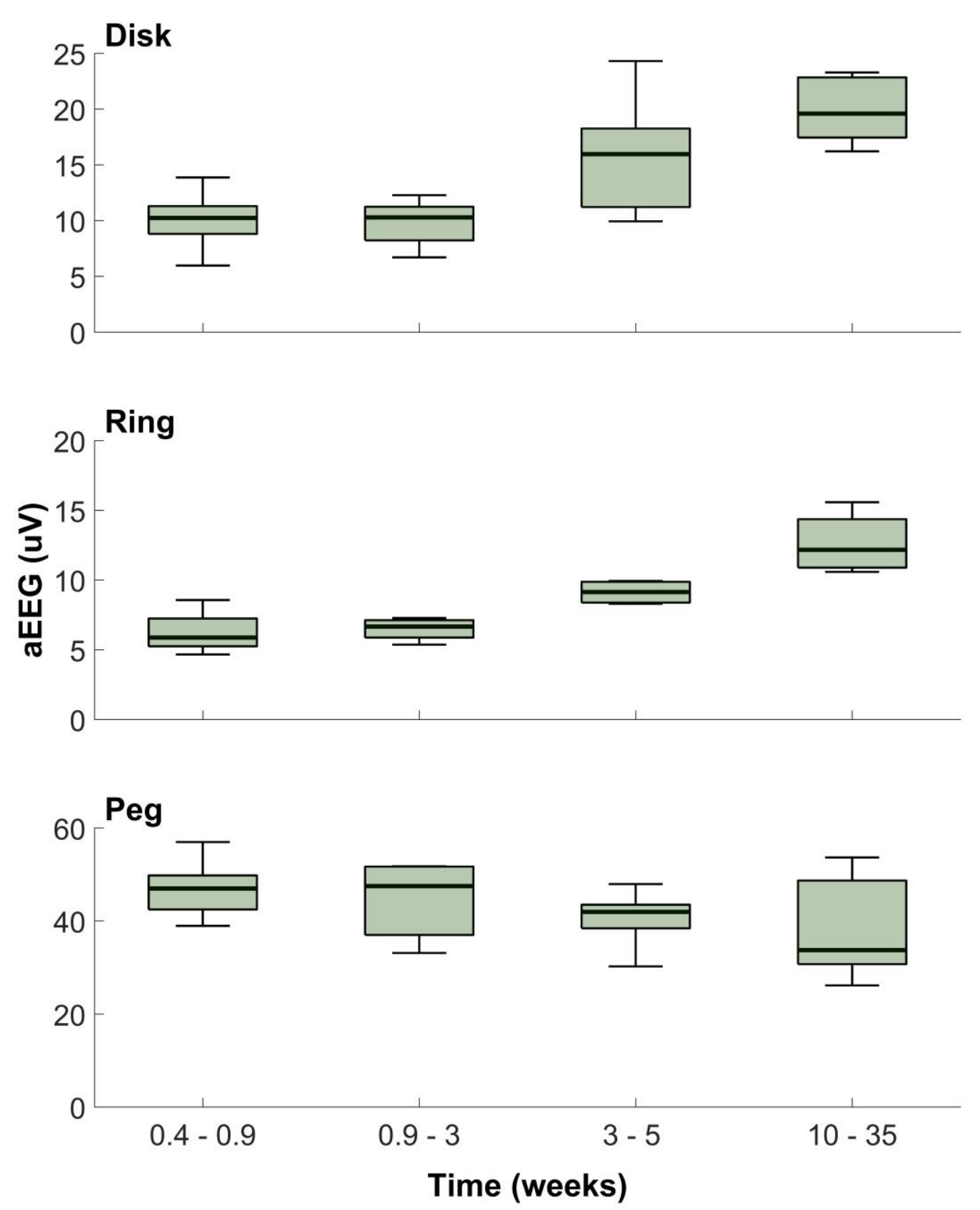

Figure 6

Amplitude-integrated electroencephalography (aEEG) disk, ring, and peg sub-scalp electrodes. recorded from 5 animals (S1 to S5) over about 3 months. On each box, the central bold mark is the median, the edges of the box are the 25th and 75th percentiles, and the whiskers extend to the most extreme data points. Friedman chi-square test indicated non-significant subject and time effects ( $p>0.05$ in both cases). 


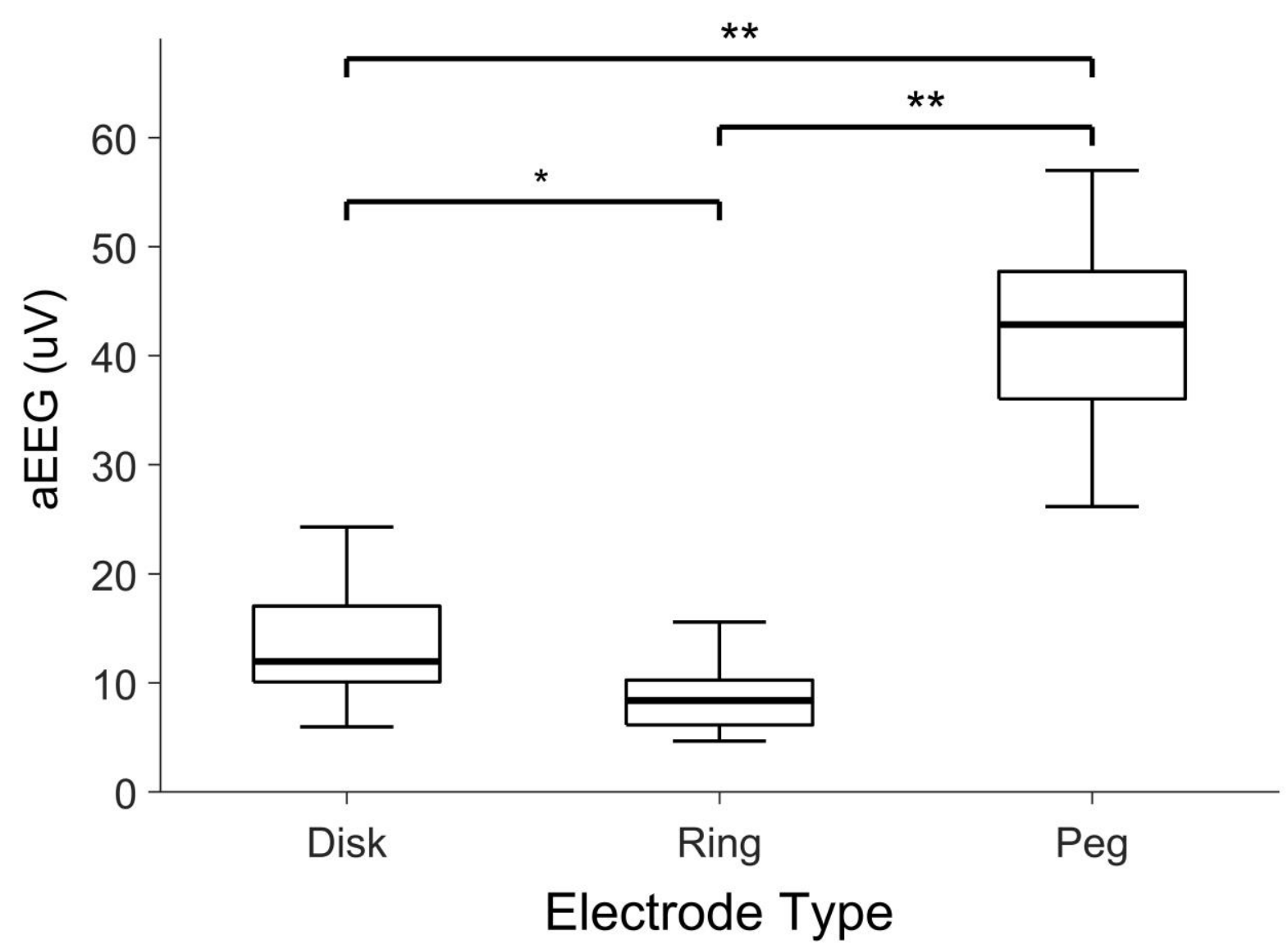

\section{Figure 7}

Distribution of amplitude-integrated electroencephalography (aEEG) for all sheep plotted for each electrode type. On each box, the central mark is the median, the edges of the box are the 25 th and 75th percentiles, and the whiskers extend to the most extreme data points. KruskalWallis with Bonferroni correction (59 degrees of freedom) indicated significant differences between electrodes $\left({ }^{*} \mathrm{p}<0.05\right.$ for disk and ring; ${ }^{* *} \mathrm{p}<0.01$ for the peg) 


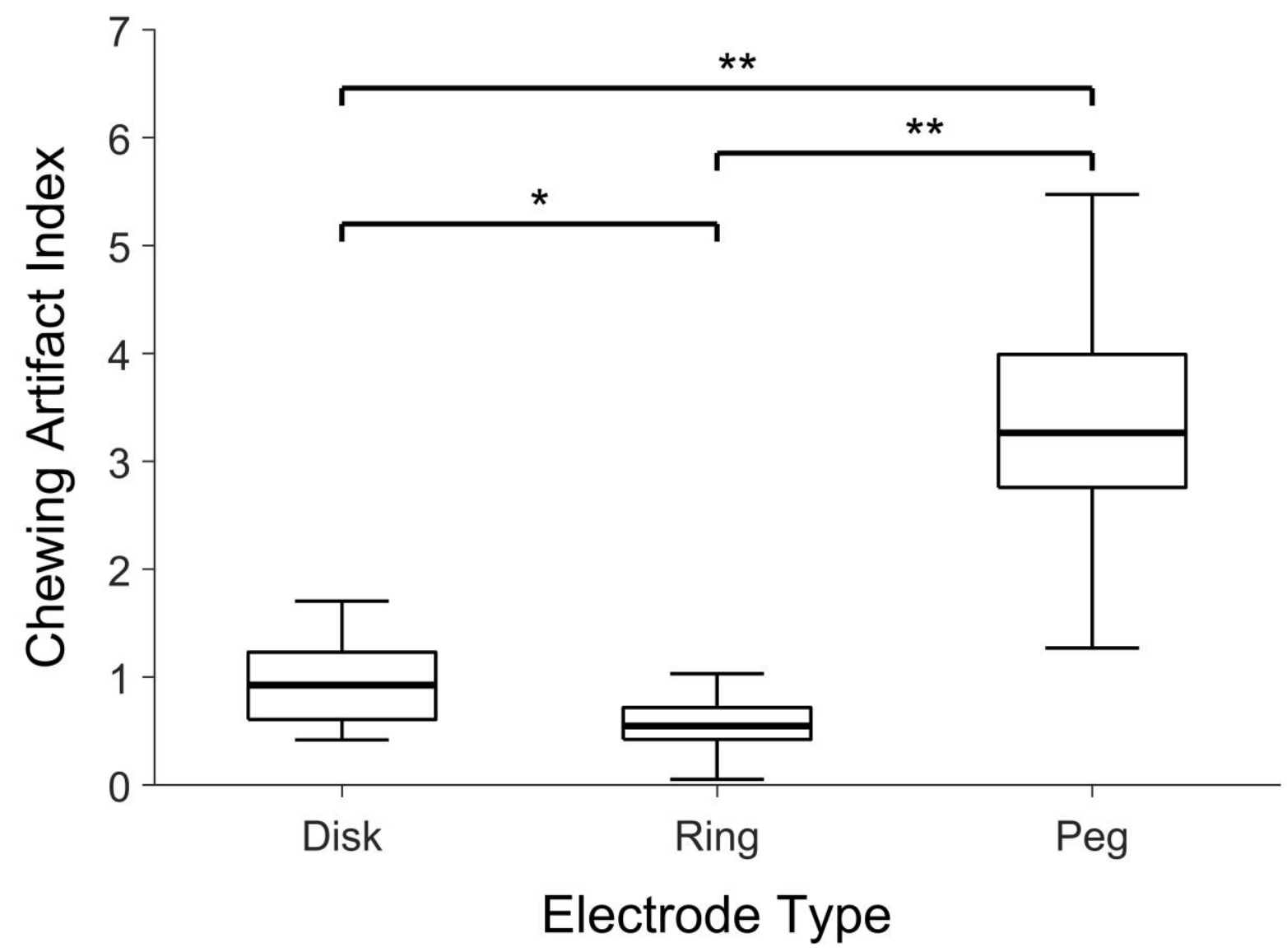

Figure 8

Chewing artifact index, which is the ratio between median amplitude of slow wave EEG and chewing artifact, for all subjects and electrode types. On each box, the central mark is the median, the edges of the box are the 25th and 75th percentiles, and the whiskers extend to the most extreme data points. Kruskal-Wallis with Bonferroni correction (75 degrees of freedom) indicated significant differences between electrodes $\left({ }^{*} \mathrm{p}<0.05\right.$ for disk and ring; ${ }^{* *} \mathrm{p}<0.01$ for the peg). 

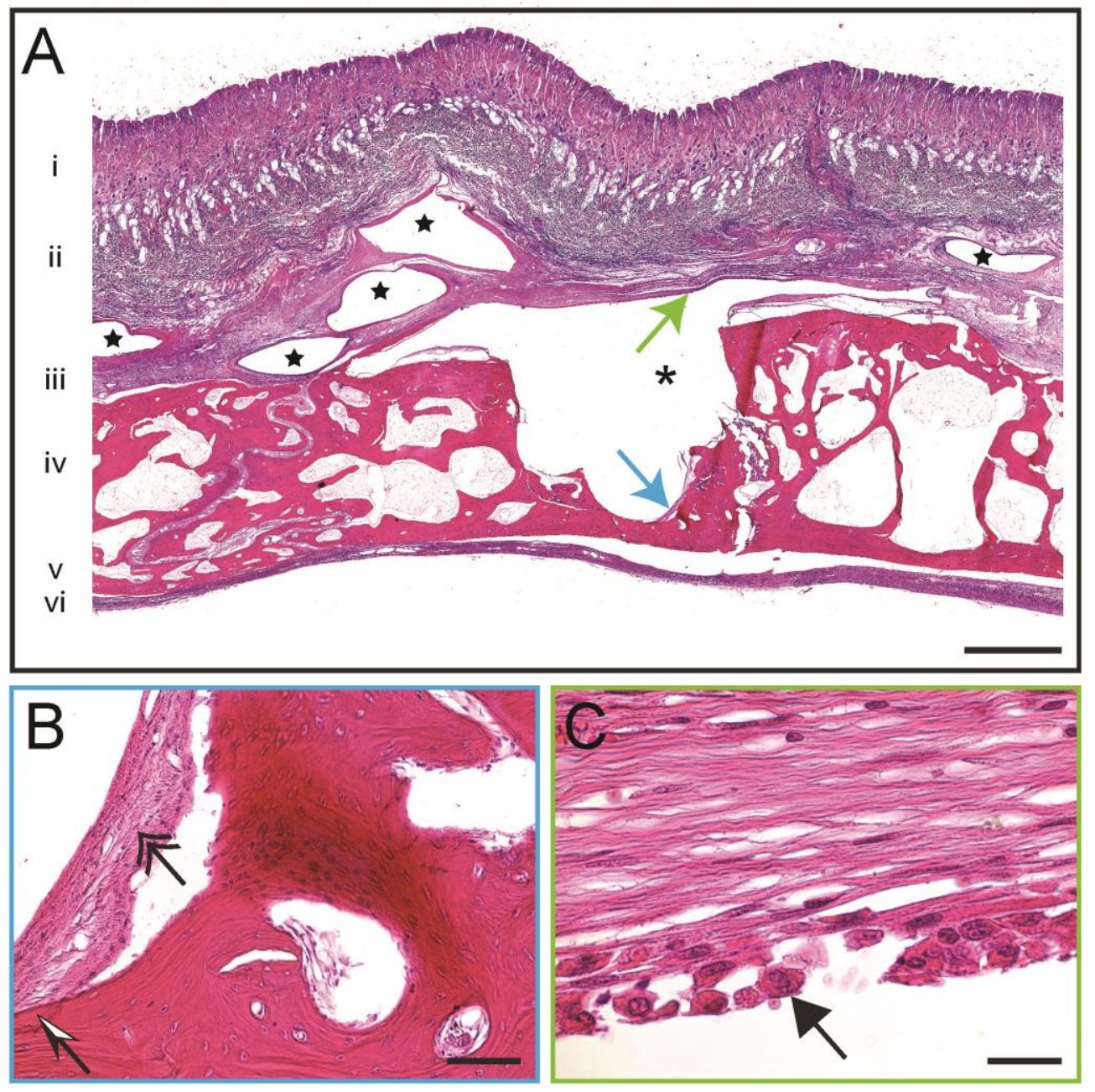

\section{Figure 9}

Haematoxylin and eosin (H\&E) stained photomicrographs illustrating the histology following chronic implantation of a peg electrode, taken from $5 \mu \mathrm{m}$ thick paraffin-embedded tissue sections. A. Low power photomicrograph (x2.5) showing the: layers of the skin (i); loose connective tissue (ii); and pericranium (iii). At the implant site a thin fibrotic capsule was observed surrounding the silicone cap of the electrode (green arrow). Cavities were noted where electrode wiring was routed (stars). In other respects, the skin overlying the electrode was unremarkable compared with distal skin. The space in the bone occupied by the peg electrode (iv) can be clearly distinguished (asterisk). The bone immediately adjacent to the electrode cavity was unremarkable in comparison to distal bone. In this case the tip of the electrode was positioned in the lower table of the skull (v). The lowest layer depicted is the dura mater (vi) which has been artifactually displaced in some places. Scale bar $=1 \mathrm{~mm}$. B. Higher power photomicrograph (x20) of the region indicated by the blue arrow in panel A, depicting the skull adjacent to the side of the peg implant. Down-growth of pericranium-like connective tissue was observed on the inner wall of the electrode cavity (double arrow). In places, this tissue lining was 
artifactually displaced - likely during removal of the peg electrodes prior to embedding in paraffin for sectioning (pied arrow). Scale bar $=100 \mu \mathrm{m}$. C. High power photomicrograph (x80) of the region indicated by the green arrow in panel A, depicting a thin fibrotic encapsulation of the implant. The tissue response was slight but most prominent at the superior surface of the silicone cap. There was a minimal inflammatory reaction with multinucleated cells (arrow) of approximately one to three cell layers in thickness. Superior to this encapsulation was loose connective tissue. Scale bar $=20 \mu \mathrm{m}$.
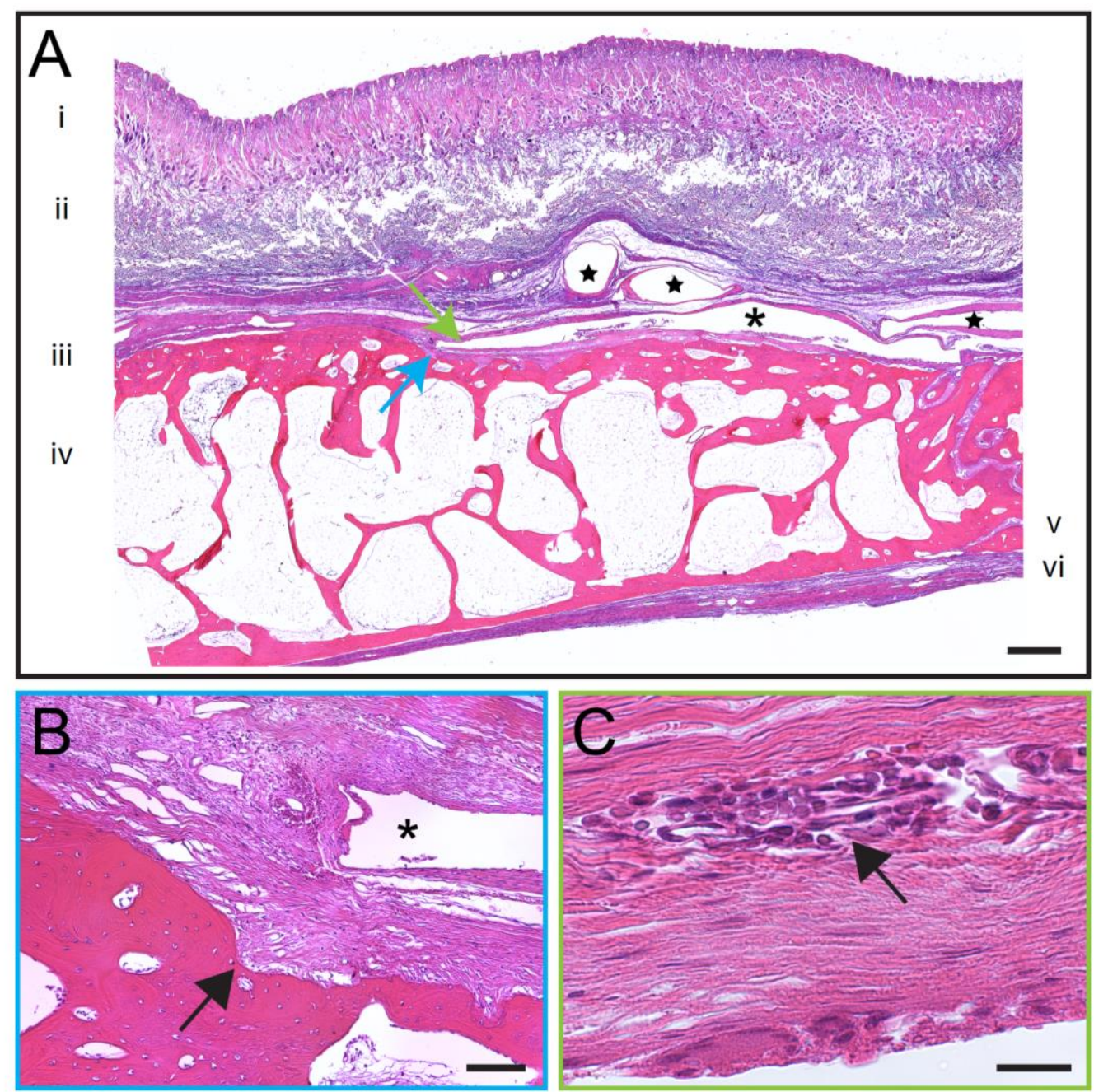

\section{Figure 10}

Haematoxylin and eosin (H\&E) stained photomicrographs illustrating the histology following chronic implantation of a disk electrode, taken from $5 \mu \mathrm{m}$ thick paraffin-embedded tissue sections. A. Low power photomicrograph (x2.5) showing the: layers of the skin (i); loose connective tissue (ii); and pericranium (iii). At the implant site a thin fibrotic capsule was observed surrounding the disk electrode cavity (asterisk). Cavities were noted where electrode 
wiring was routed (stars). The outer table of the bone (iv) immediately underlying the disk was eroded. The lower table of the skull (v) and dura mater (vi) were unremarkable. Scale bar $=1$ $\mathrm{mm}$. B. Higher power photomicrograph (x10) of the region indicated by the blue arrow in panel A, depicting the edge of the disk electrode and neighboring tissue. The asterisk indicates the original location of the disk electrode. The outer table of the bone immediately underlying the disk electrode was eroded, most likely due to pressure effect. The arrow indicates the junction of eroded and non-eroded outer table region. Scale bar $=100 \mu \mathrm{m}$. C. High power photomicrograph (x63) of the region indicated by the green arrow in panel A, depicting a thin fibrotic encapsulation of the implant. There was a minimal inflammatory reaction with uni- and multinucleated histiocytic cells (arrow). Scale bar $=20 \mu \mathrm{m}$.

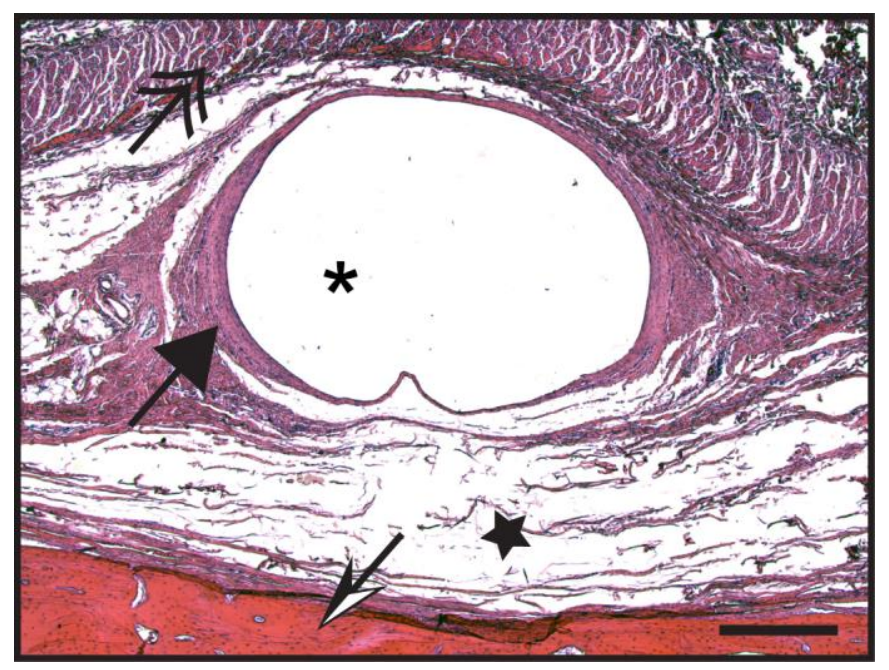

\section{Figure 11}

Haematoxylin and eosin (H\&E) stained photomicrograph (x10) illustrating the histology following chronic implantation of a ring electrode, taken from $5 \mu \mathrm{m}$ thick paraffin-embedded tissue sections. The region surrounding a ring electrode cavity (asterisk) is surrounded by a thin fibrous capsule partially lined by uni- and multi-nucleated histiocytic cells (arrow). The scale bar is $500 \mathrm{um}$. The double arrow shows the overlying muscle layer that seems to have been adversely affected by the processing. The pied arrow shows the underlying bone. The star shows some loose connective tissue that likely to have been artifactually separated. 


\section{University Library}

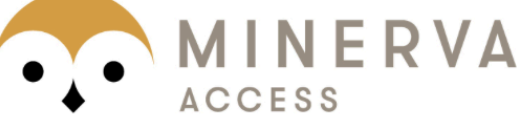

A gateway to Melbourne's research publications

Minerva Access is the Institutional Repository of The University of Melbourne

Author/s:

Benovitski, YB;Lai, A;McGowan, CC;Burns, O;Maxim, V;Nayagam, DAX;Millard, R;Rathbone, GD;le Chevoir, MA;Williams, RA;Grayden, DB;May, CN;Murphy, M;D'Souza, WJ;Cook, MJ;Williams, CE

Title:

Ring and peg electrodes for minimally-Invasive and long-term sub-scalp EEG recordings

Date:

2017-09-01

Citation:

Benovitski, Y. B., Lai, A., McGowan, C. C., Burns, O., Maxim, V., Nayagam, D. A. X., Millard, R., Rathbone, G. D., le Chevoir, M. A., Williams, R. A., Grayden, D. B., May, C. N., Murphy, M., D'Souza, W. J., Cook, M. J. \& Williams, C. E. (2017). Ring and peg electrodes for minimally-Invasive and long-term sub-scalp EEG recordings. Epilepsy Research, 135, pp.29-37. https://doi.org/10.1016/j.eplepsyres.2017.06.003.

Persistent Link:

http://hdl.handle.net/11343/242236 